\title{
Vasopressin acts as a synapse organizer in limbic regions by boosting PSD95 and GluA1 expression
}

\author{
Running title: AVP modulates PSD protein expressions
}

\author{
Limei Zhang ${ }^{1,3 c}$, Teresa Padilla-Flores ${ }^{1,3}$, Vito S. Hernández ${ }^{1,3}$, Elba Campos-Lira ${ }^{1}$, Mario A. Zetter ${ }^{1}$, \\ Laura I. Escobar ${ }^{1}$, Lee E. Eiden ${ }^{2}$ \\ ${ }^{1}$ Dept. Physiology, School of Medicine, National Autonomous University of Mexico, Mexico \\ 2 Section on Molecular Neuroscience, NIMH-IRP, NIH, Bethesda, MD, USA \\ ${ }^{3}$ These authors contributed equally to this work \\ ${ }^{c}$ Corresponding author: Prof. Limei Zhang, Department of Physiology, School of Medicine, National Autonomous \\ University of Mexico, Av. Universidad 3000, CDMX, 04510, Mexico.
}

\section{Abstract}

Hypothalamic arginine vasopressin (AVP)-containing magnocellular neurosecretory neurons (AVPMNN) emit collaterals to synaptically innervate limbic regions influencing learning, motivational behavior and fear responses. The purpose of the present work is to characterize the dynamics of expression changes of two postsynaptic density (PSD) proteins, AMPAR subunit GluA1 and PSD scaffolding protein 95 (PSD95), which are known to be key determinants for synaptic strength, in response to in vivo and ex vivo manipulations of AVPMNN neuronal activation state, or exposure to exogenous AVP, metabolites and some signaling pathway inhibitors. Both long term water deprivation in vivo, which powerfully upregulates AVPMNN activity, and exogenous APV application ex vivo in brain slices, increased GluA1 and PSD95 expression and enhanced neuronal excitability in ventral hippocampal CA1 pyramidal neurons. Involvement of PI3K signaling in AVP-dependent plasticity is suggested by blockade of both AVP-induced protein up-regulation and enhanced neuronal excitability by the PI3k blocker wortmannin in hippocampal slices. We interpret these results as part of vasopressin's central effects on synaptic organization in limbic regions modulating the strength of a specific set of synaptic proteins in hypothalamic-limbic circuits.
\end{abstract}

Keywords: water deprivation, Western blot, whole-cell patch-clamp, ventral hippocampus, central amygdala, habenula, wortmannin.

Supported by grants: UNAM-DGAPA-PAPIIT-IN216918 \& CONACYT-CB-238744. 


\section{AVP modulates PSD protein expressions}

\section{Introduction}

The neurohormone arginine vasopressin (AVP, also called antidiuretic hormone, ADH) is mainly synthesized in the hypothalamus and released by the posterior hypophysis as the key regulator of body water homeostasis (Boone and Deen 2008). The AVP magnocellular neurosecretory neurons (AVPMNN), together with those for oxytocin, were the first peptidergic systems to be described in the mammalian brain (Bargmann and Scharrer 1951). Release of AVP from both posterior pituitary lobe and median eminence, as well as AVPMNN somato-dendritic release within the hypothalamus itself (Pow and Morris 1989, Brown, Ludwig et al. 2020) helps to control water homeostasis. AVP also acts as a neurotransmitter within the brain, via Gray type I excitatory synapses (Peters 1970) in amygdala, hippocampus and habenula (Cui, Gerfen et al. 2013, Zhang and Hernandez 2013, Hernandez, Vazquez-Juarez et al. 2015, Hernandez, Hernandez et al. 2016, Zhang, Hernandez et al. 2016, Zhang, Hernandez et al. 2018, Zhang, Hernandez et al. 2020). The development from an endocrine homeostatic role for vasopressin, to a dual endocrine-neurotransmitter one, parallels the evolutionary emergence of allostatic (anticipatory) control mechanisms, alongside those for homeostasis, throughout the vertebrata and most extensively studied in mammalia (Zhang and Eiden 2019).

David de Wied in the 1970 s pioneered studies suggesting that vasopressin may act as a neurotransmitter or neuromodulator at synapses within the brain cognitive/emotional control centers (De Wied 1971, de Wied 1976). Shortly after, a series of studies published in the 1980s and early 1990s showed that sub-micromolar concentrations of vasopressin could, in brain slices ex vivo, powerfully and reversibly increase the rate of firing of neurons in the CA1 areas of rat hippocampal slices and that this effect could be fully antagonized by an anti-vasopressor vasopressin analogue (Muhlethaler, Dreifuss et al. 1982). Hippocampal neurons obtained from Brattleboro rats, deficient in AVP, were also excited by exogenous vasopressin (Muhlethaler, Dreifuss et al. 1982). AVP-induced potentiation of the excitatory postsynaptic potential (EPSP) was long-lasting, persisting after removal of AVP from the perfusion medium (Chen, Diaz Brinton et al. 1993). The AVP-induced sustained increase of EPSP is known as long-term vasopressin potentiation (Chen, Diaz Brinton et al. 1993). Both AVP and its metabolite AVP(4-8) were found to elicit a long-lasting enhancement of hippocampal excitability, mostly in neurons within the ventral hippocampus (vHi) (Chen, Diaz Brinton et al. 1993, Urban 1998, Chepkova, Kapai et al. 2001, Dubrovsky, Tatarinov et al. 2003).

Despite the plethora of observations detailing AVP actions at hippocampal and other cognitive hubs in the CNS (vide supra), the cellular mechanisms by which AVP potentiates neuronal excitability are not known. AVP-containing dense core vesicles (AVP+ DCV) have been observed at excitatory synaptic active zones, docked onto the presynaptic membrane, suggesting that vasopressin modulation of neurotransmission may occur at the level of the synapse itself within limbic regions such as hippocampus (Zhang and Hernandez 2013), amygdala (Hernandez, Hernandez et al. 2016), and lateral habenula (Zhang, Hernandez et al. 2016) (Figure 1). 


\section{AVP modulates PSD protein expressions}

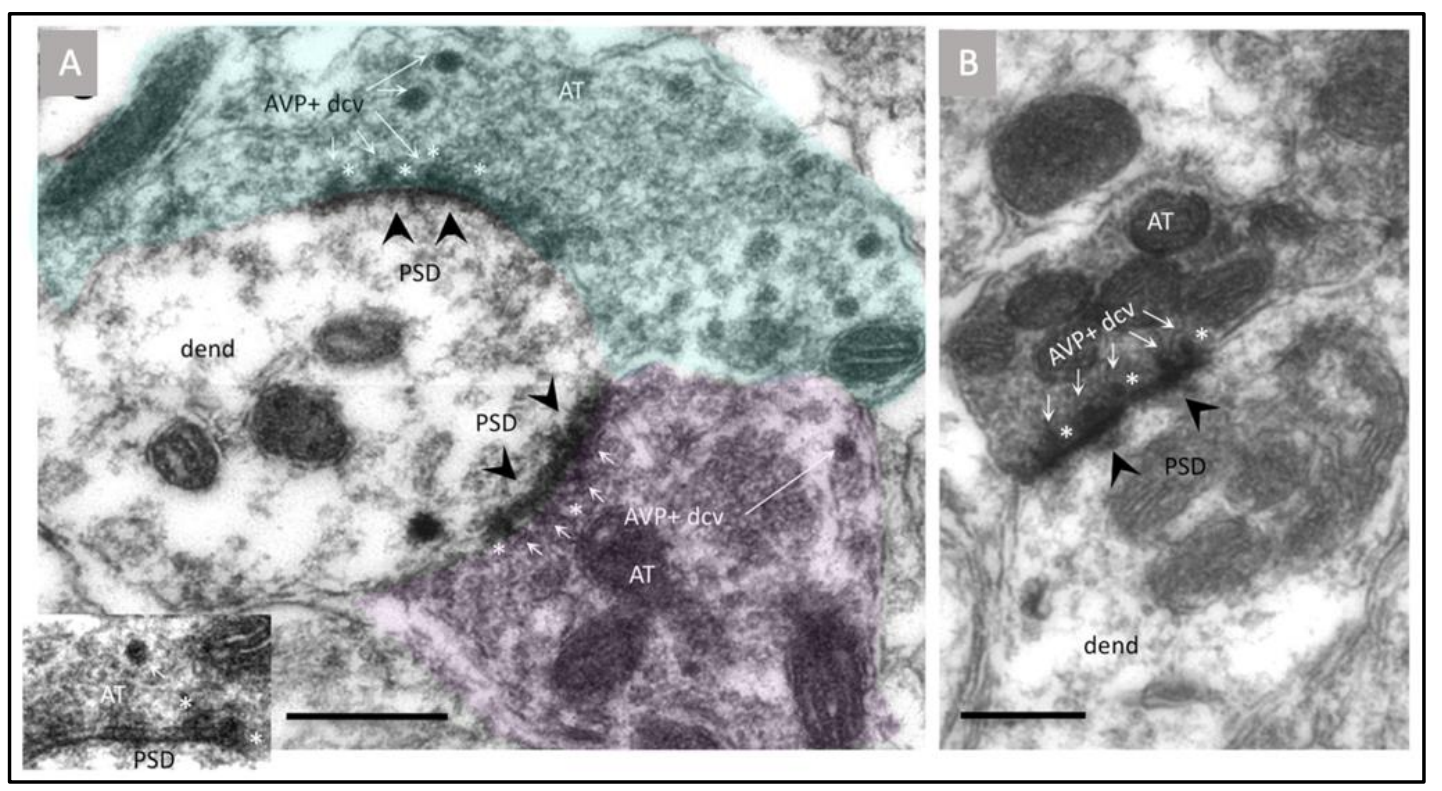

Figure 1. Ultrastructural examples showing morphological evidence that AVP could directly modulate the synaptic function. A: two Gray type I synapses made by two AVP-immunopositive $(A V P+)$ axon terminals (AT, green and pink) contain AVP+ dense core vesicles (DCV, white arrows), onto an unlabeled dendrite (dend). Inset shows higher magnification of four AVP+ DCV docked on the active zone of the presynaptic membrane. Postsynaptic density (PSD), a morphological feature of glutamatergic synapse, is indicated by black arrowhead. Sample was taken from rat lateral habenula. B: analogue notations to A, Sample taken from rat ventral hippocampus. Panel A is modified from (Zhang, Hernandez et al. 2016) (Creative Common Attribution License holder) and panel B is taken from (Zhang and Hernandez 2013) with permission from the original publisher. Scale bars: $500 \mathrm{~nm}$.

With this background, we asked whether AVP could participate in the modulation of synapse strength by dynamically modifying the protein composition of the postsynaptic density (PSD). We characterized the dynamics expression changes of two postsynaptic density (PSD) proteins, AMPAR subunit GluA1 and PSD scaffolding protein 95 (PSD95), which are known to be key determinants for synaptic strength, under in-vivo and ex-vivo conditions with AVP system upregulation or application.

\section{Materials and methods}

Animals

Adult male Wistar rats of 250 +/- $20 \mathrm{~g}$ and males at postnatal day 30 (PND30), were used in this study. Rats were obtained from local animal vivarium and were housed three per cage under controlled temperature and illumination $(12 \mathrm{~h} / 12 \mathrm{~h})$ with water and food chow ad libitum unless otherwise indicated. All animal procedures were approved by the local research ethics supervision commission (license number: CIEFM-062-2016). 


\section{AVP modulates PSD protein expressions}

\section{Chemicals and primary antibodies used in this study}

Primary antibodies and their dilutions used in this study: mouse monoclonal anti-PSD95 (UC Davis, clone K28/74, $1 \mathrm{mg} / \mathrm{mL}, 1: 1000, C A$, USA, http://neuromab.ucdavis.edu/datasheet/K28_43.pdf), mouse monoclonal anti-PSD-95 (7E3) (Santa Cruz, sc-32290, $200 \mu \mathrm{g} / \mathrm{ml}$, CA, USA; https://www.scbt.com/es/p/psd-95-antibody-7e3); guinea pig polyclonal anti-GluR1 (Frontiers Institute, GluA1-GP-Af380, 200 $\mathrm{g} / \mathrm{mL}$, 1:250, Japan, https://nittobo-nmd.co.jp/pdf/reagents/GluA1.pdf). Vasopressin for clinical use (Arterina $20 \mathrm{UI} / \mathrm{ml}$ PiSA, Mexico) and kindly donated by Prof. Maurice Manning (MK I-23) was used. Customer designed vasopressin fragments AVP4-8 (P1601291), AVP4-9

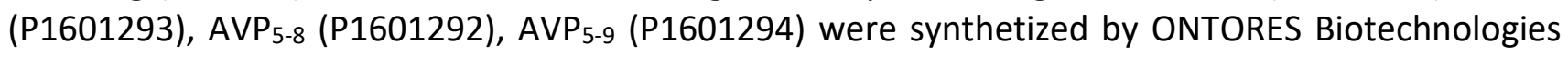
(Zhejiang, China). Inhibitors for the following signaling molecules/pathways, phosphoinositide 3kinase (PIP3K)/protein kinase B (PKB or Akt), protein kinase A (PKA), protein kinase C (PKC), mitogenactivated protein kinase (MAPK) were used, i.e. wortmannin, Sigma-Aldrich, Cat 19545-26-7, Darmstadt, Germany), H89 (Tocris, Cat. 2910, Bristol, UK), Go 6976, (Calbiochem, Cat. 365250, La Jolla, USA) and U0126 (Cat 1144, Bristol, UK), respectively.

\section{Experimental design}

Three sets of experiments (noted as A, B and C) were performed for this study. Experiment $A$ evaluated changes in PSD95 and GluA1 protein expression levels in an in vivo water deprivation treatment model, in which the variables were different time-points of the treatment. With this physiological treatment, the hypothalamic vasopressin system is known to be upregulated in a relatively selective manner, even before a significant disruption of the body's water homeostasis occurs (Dunn, Brennan et al. 1973). Experiment B aimed to determine if exogenous AVP and some of its metabolites played a direct role in the detected changes in an ex vivo slice model. Experiment $C$ evaluated in the ventral hippocampus the time-course for such changes following AVP exposure, the effect of AVP in the excitability of pyramidal neurons and the possible participation of the intracellular pathways, previously reported to induce synthesis of PSD95.

\section{Water deprivation treatment and unit-subject definition for Western blot assay}

Twenty-four experimental subjects were divided in four times points: baseline or control (water and food ad libitum), 24 hours of water deprivation (24h WD), 48 hours of water deprivation (48h WD) and 24 hours of water restoration after 48h WD (48h WD $+24 \mathrm{~h} \mathrm{R})$. To show the dynamic change of PSD95 and GluA1 as a function of the treatment, and to assure that the samples for each region possessed anatomical precision and sufficient protein amounts, we pooled tissues from 2 rat brain as one subject at a given time-point. Hence, each time-point has $n=3$ subjects. Here we introduce the unit-subject concept that is defined as protein samples of a given anatomical region coming from 8 rats, distributed in 4 time-points, which were assayed in a same western blot. Three complete blots per each region ( $n=3$-unit subjects) contributed to the data set of this study that we presented entirely in the Fig. 2 to allow the readers to have direct observation, beside of the numerical data and statistical analysis. 


\section{AVP modulates PSD protein expressions}

\section{Sample obtention and Western blot procedure}

After the corresponding treatment periods (baseline, 24h WD, 48h WD and 48h WD + 24h R), rats were deeply anesthetized under an overdose of sodium pentobarbital $(63 \mathrm{mg} / \mathrm{kg}$, Sedalpharma, México) and killed by rapid decapitation using a rodent guillotine. Fresh brain tissue was removed quickly and dissected on ice-cooled saline $(0.9 \%)$ solution. Brains were coronally blocked from optic chiasm (around Bregma $0.00 \mathrm{~mm}$ ) to posterior Bregma $-7.00 \mathrm{~mm}$ and put on a tissue chopper for thick slice preparation (0.5 $\mathrm{mm}$ thickness). Segments of brain sections containing central amygdala, habenula and ventral hippocampus ( $\mathrm{vHi}$ ) and primary visual cortex (V1, served as a negative control region since no direct innervations to this region from hypothalamic AVPMNN system have been reported), were obtained under a stereoscopic microscope using microdissection tools. Each sample of a given brain region interested in this study was pooled from the same region from 2 rats, in order to have enough brain tissue for immunoblotting while not sacrificing anatomical precisions. Dissected brain regions were sonicated in ice-cold extraction buffer: $50 \mathrm{mM}$ Tris- $\mathrm{HCl} \mathrm{pH} \mathrm{7.4,} \mathrm{8.5 \%} \mathrm{sucrose,} 2$ mM EDTA, supplemented with proteases and phosphatases inhibitors $(8 \mu \mathrm{g} / \mathrm{mL}$ aprotinin, $10 \mu \mathrm{g} / \mathrm{mL}$ leupeptin, $4 \mu \mathrm{g} / \mathrm{mL}$ pepstatin A, $500 \mu \mathrm{M}$ AEBSF, $5 \mathrm{mM}$ benzamidine, $20 \mathrm{mM}$ ß-glycerophosphate, 10 $\mathrm{mM}$ sodium fluoride, $1 \mathrm{mM}$ sodium orthovanadate). Brain homogenates were assayed for protein using the BCA method (Pierce, Thermo Scientific cat\# 23225). For SDS-PAGE, homogenates (15 $\mu \mathrm{g}$ total protein) were mixed with loading buffer added with ß-mercaptoethanol and boiled for $3 \mathrm{~min}$. Samples were separated in $10 \%$ acrylamide gels and transferred to $0.2 \mu \mathrm{m}$ nitrocellulose membrane (Amersham, GE cat\# 10600006). Blots were blocked for $1 \mathrm{~h}$ at room temperature (RT) in Tris-buffered saline with $0.05 \%$ Tween-20 (TBST) containing $5 \%$ blotting-grade dry milk (Biorad cat\# 170-6404), incubated overnight with primary antibody diluted (dilutions above mentioned) in blocking buffer at $4^{\circ} \mathrm{C}$, washed $3 \times 10 \mathrm{~min}$ in TBST, incubated with secondary antibody horseradish peroxidaseconjugated (1:5000, Jackson ImmunoResearch) for $1 \mathrm{hr}$. at RT, and washed 3x $10 \mathrm{~min}$ in TBST. Membranes were visualized with luminol substrate by enhanced chemiluminescence (Mruk and Cheng 2011). PSD95 and GluA1 were evaluated in the same gel, for that purpose membranes were incubated in stripping buffer (0.2 M glycine pH 2.2, $0.1 \%$ SDS, $1 \%$ Triton X-100) for 2x 15 min at RT, washed and re-probed using the above protocol. For antibodies and their dilutions used in this study please see section 2.2 .

The densitometric analysis was performed using Image J (NIH, Bethesda, USA) according to Davarinejad H. (www.yorku.ca/yisheng/Internal/Protocols/ImageJ.pdf). Blots were scanned with ChemiDoc (BioRad, USA). The images were converted to grayscale. The mean gray value of a unified area was determined using the rectangular selection tool. The same rectangle was placed on protein bands areas (e.g. PSD95, GluA1 and GAPDH), as well as on the backgrounds immediate above or below each band. The final value of each band was the result of the signal after subtracting the background value. The ratio of signals of PSD95 or GluA1 divided by GAPDH (the loading control) was obtained and normalized with respect to the average value of corresponding control bands and are expressed as relative expression of (GluA1 or PSD95) / (GAPDH).

Hippocampal acute slice pharmacology and electrophysiology with exogenous AVP and metabolites incubation

Six rats at PND30 were deeply anesthetized using an overdose of sodium pentobarbital $(63 \mathrm{mg} / \mathrm{kg}$, Sedalpharma, México) and killed by rapid decapitation using a rodent guillotine. Fresh brain tissue 


\section{AVP modulates PSD protein expressions}

was removed quickly and blocked on ice-cooled saline (0.9\%) solution. Brain horizontal slices of 300 $\mu \mathrm{m}$ thickness containing the ventral hippocampus were obtained using a Leica VT 1000S vibratome, in ice-cold cutting solution containing the following (in $\mathrm{mM}$ ): $75 \mathrm{NaCl}, 2.5 \mathrm{KCl}, 25 \mathrm{NaHCO}_{3}, 1.25$ $\mathrm{NaH}_{2} \mathrm{PO}_{4}, 25$ glucose, $0.1 \mathrm{CaCl}_{2}$, and $6 \mathrm{MgCl}_{2}$, and 50 sucrose bubbled with a mixture of $5 \% \mathrm{CO}_{2}$ and $95 \% \mathrm{O}_{2}, \mathrm{pH} 7.4$ (Zhang, Hernandez et al. 2016). The slices were then transferred to artificial cerebrospinal fluid (ACSF) containing the following (in mM): $125 \mathrm{NaCl}, 2.5 \mathrm{KCl}, 25 \mathrm{NaHCO}_{3}, 1.25$ $\mathrm{NaH}_{2} \mathrm{PO}_{4}, 25$ glucose, $2 \mathrm{CaCl}_{2}$, and $2 \mathrm{MgCl}_{2}$ bubbled with a mixture of $5 \% \mathrm{CO}_{2}$ and $95 \% \mathrm{O}_{2}, \mathrm{pH} 7.4$ to stabilize, at room temperature for $30 \mathrm{~min}$. After this time, the slices were incubated with corresponding reagents for the given time periods specified at $37^{\circ} \mathrm{C}$ in the same artificial cerebrospinal fluid (ACSF).

To determine the AVP's role in the upregulated expression of GluA1 and PSD95, observed in vivo, we evaluated the in vitro effects of exogenous AVP and AVP fragments applied to the slices in the incubation medium. Each rat provided six sections (three per hemisphere containing the ventral hippocampus (vHi) for the 6 experimental groups: Ctrl; $100 \mathrm{nM}$ AVP (Arterina, Pisa 20 I.U. per ml); 100 nM AVP 4-8 (GIn-Asn- Cys-Pro-Arg-NH); 100 nM AVP 4-9 (Gln-Asn-Cys-Pro-Arg-Gly-NH); 100 nM AVP 5-8 (Asn-Cys-Pro-Arg-NH) and 100 nM AVP 5-9 (Asn-Cys-Pro-Arg-Gly-NH). The incubation time was $2 \mathrm{hr}$. at $37^{\circ} \mathrm{C}$. One of the $6 \mathrm{vHi}$ slices from one rat was pooled with another in order to obtain sufficient amount of proteins for Western blot assay.

To determine the time course of exogenous AVP application in the changes observed in the previous experiment, we assayed the PSD95 and GluA1 expression under exogenous AVP application (ACSF alone, or ACSF with 100nM AVP (from Prof. Maurice Manning's lab) at four time-points: baseline, $5 \mathrm{~min}, 60 \mathrm{~min}$ or $120 \mathrm{~min}$. We used slices from 4 rats that each subject provided four $\mathrm{vHi}$ slices for all time-points and slices from 2 rats were pooled to run in the same blot.

To determine the effects of AVP on the excitability of pyramidal neurons in area CA1 of vHi, we collected $\mathrm{vHi}$ horizontal slices as above mentioned, and performed whole-cell patch clamp recordings in current clamp mode, using borosilicate glass pipettes with a resistance of 6-8 $\mathrm{M} \Omega$ filled with the following intracellular solution (in $\mathrm{mM}$ ): $122 \mathrm{~K}$-Gluconate, $5 \mathrm{NaCl}, 10 \mathrm{HEPES}, 2 \mathrm{MgCl} 2,1 \mathrm{EGTA}$, $0.3 \mathrm{CaCl}_{2}, 5 \mathrm{Na}_{2} \mathrm{ATP}, 0.4 \mathrm{Na}_{3} \mathrm{GTP}$ and $1 \mathrm{mg} / \mathrm{ml}$ of neurobiotin. Action potentials induced by the injection of 500 millisecond square current pulses of 50,100, 150 and 200 pA were counted 1) baseline, 2) after 45-60 min of 100nM AVP in ACSF perfusion ("AVP") and 3) after 30 min elapsed from the time AVP was washed from the medium ("Wash"). For each neuron the protocol was run five times in each of the conditions and the average number of spikes elicited by each current step was obtained and graphed. The current vs firing rate (I-FR) graphs were calculated with the values obtained from 6 neurons. The resting membrane potential (RMP) was obtained when no current step was applied. The input resistance was calculated with the voltage change induced by a -50 pA pulse of current by using Ohm's law. After the recordings the slices were fixed in $4 \%$ paraformaldehyde in PB 0.1M for 1 hour, washed thoroughly and incubated with 488-Streptavidin 1:1000 in Trisma Buffer $+0.9 \mathrm{NaCl}+$ triton $0.3 \%$ (TBST) overnight. Next day the morphology of the neuron was observed under an epifluorescence microscope to corroborate the position in CA1 pyramidal layer. Some neurons that were anatomically well conserved (soma was clearly visible, without significant edema and dendrites were well labelled) were further immunoreacted against PSD95 and observed under 


\section{AVP modulates PSD protein expressions}

confocal microscope to evaluate if there was significantly different number of PSD95 puncta after AVP incubation. Some control neurons not exposed to AVP were also filled for comparison.

The last part of this experiment was aimed to evaluate if the activation of some of the intracellular pathways known to induce the expression of PSD95 played a role in the AVP induced increase in excitability. First we tested by Western blot if the following compounds (concentrations used were stated above): wortmannin (Wm) an inhibitor of the PIP3K/AKT pathway; H89 an inhibitor of PKA ; Go-6976 an inhibitor of PKC and U0126, a MAPK inhibitor, were able to block the vasopressin induced increase in PSD95. Wortmannin was observed to reduce the AVP induced increase in PSD95 expression. Thus, we evaluated in the electrophysiological assay mentioned above if this blockade was correlated with a functional effect. For this experiment design the same current clamp protocol was used, but the initial recording was made in ACSF alone, the second recording after 45 min of exposure to $100 \mathrm{nM} A V P+10 \mathrm{mM} \mathrm{Wm}$, and the last recording was made after $30 \mathrm{~min}$ of wash.

\section{Statistical Analysis}

Quantitative results were expressed as mean \pm SEM. Differences between means were evaluated by one- or two- way ANOVA, followed by Tukey post-test or Student T-Test. All the analyses were performed using Prism (GraphPad Software, V8.0, San Diego, CA, USA). Differences were considered statistically significant at a value of $* p<0.05 ; * *<0.01 ; * * * p<0.001$.

\section{Results}

\section{Water deprivation effectively increased PSD95 and GluA1 protein expression in amygdala, lateral habenula and hippocampus}

Quantitative Western blot analysis revealed that 48 hours of water deprivation produced a significant increase in amount of protein expression of Glu1A and PSD95 in amygdala (Fig. 2As), lateral habenula (Fig. 2Bs) and ventral hippocampus (Fig. 2Cs), three regions that had previously demonstrated to receive direct innervation from the hypothalamic AVPMNN system (Cui, Gerfen et al. 2013, Zhang and Hernandez 2013, Hernandez, Hernandez et al. 2016, Zhang, Hernandez et al. 2016), while in the visual cortex, a region that AVPMNN vasopressin direct innervation has not been observed, no significant differences were observed (Fig. 2Ds). A restoration of the expression of GluA1 and PSD95 to baseline levels was noted in all groups after $24 \mathrm{~h}$ recovery from WD. Western blot sets of images " $-1,-2,-3$ " correspond to the three unit-subjects. It is worth mentioning that in order to show both the individual variability, the consistence among the three unit-subjects, and the solidity of the conclusions drawn from the experimental observations, as well as their statistics differences, we chose to show the original Western blot sets of all the tree unit-subjects in this report without editions. The mean relative expression for each treatment was normalized to the expression of the control levels. Comparisons between means was performed by one-way ANOVA (for details please see the figure legends). 


\section{A. Amygdala}

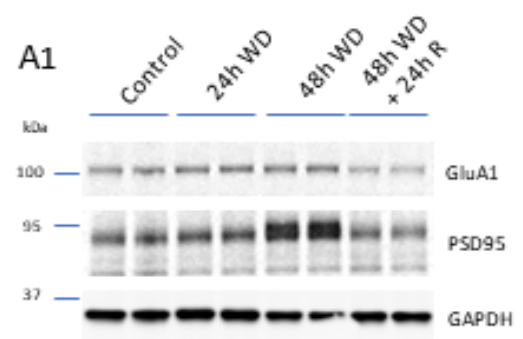

A4

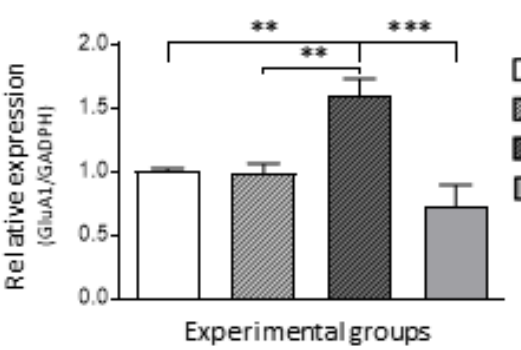

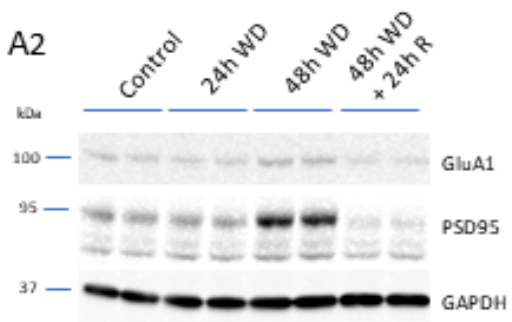

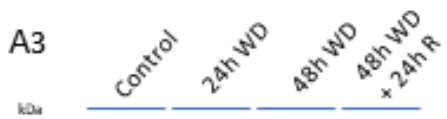

$100-\cdots+\cdots+\cdots+$ GluA1

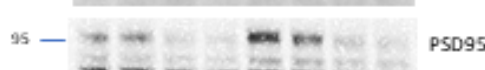

$---1-2-$

$37-\infty-\infty=6 \mathrm{GAPDH}$

\section{B. Lateral habenula}
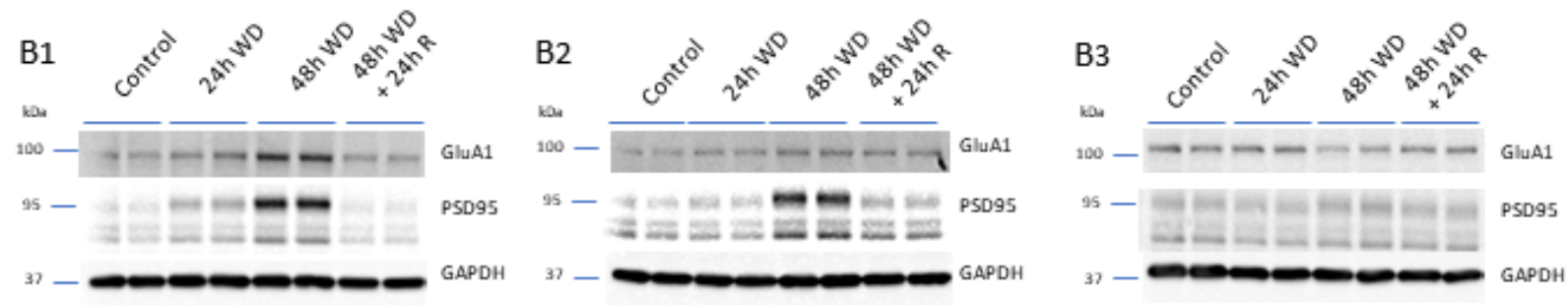

B4

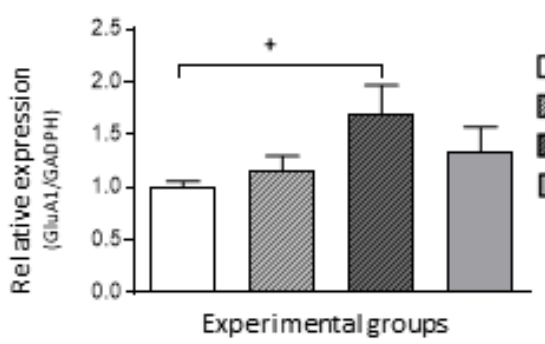

B5

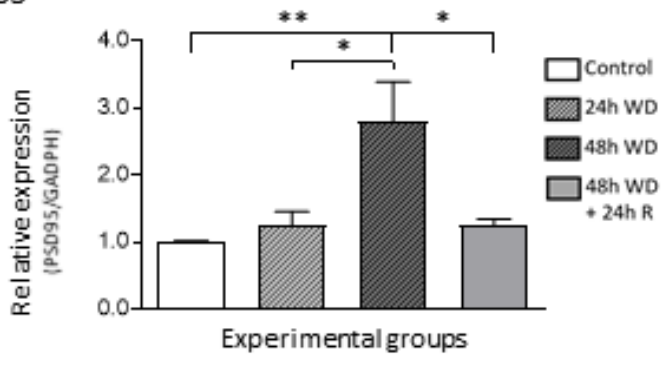




\section{Ventral hippocampus}
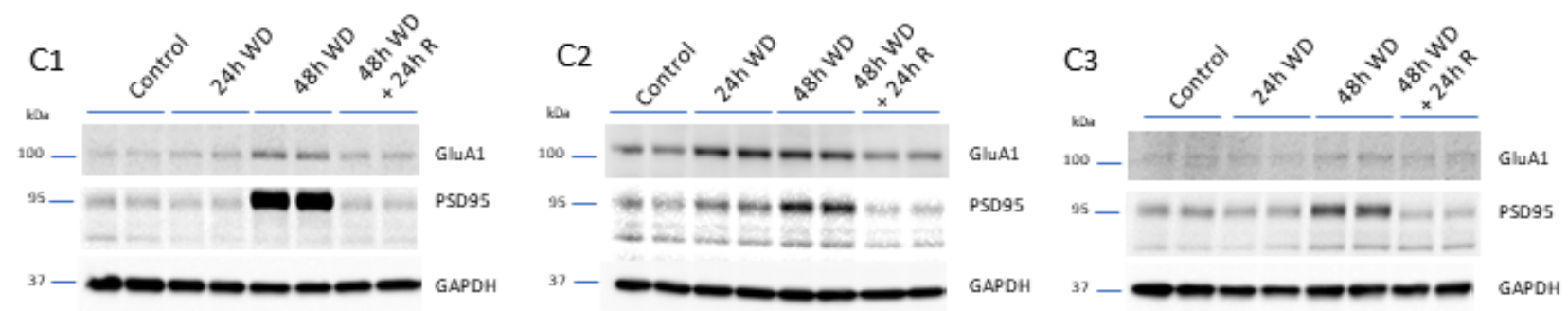

$\mathrm{C} 4$

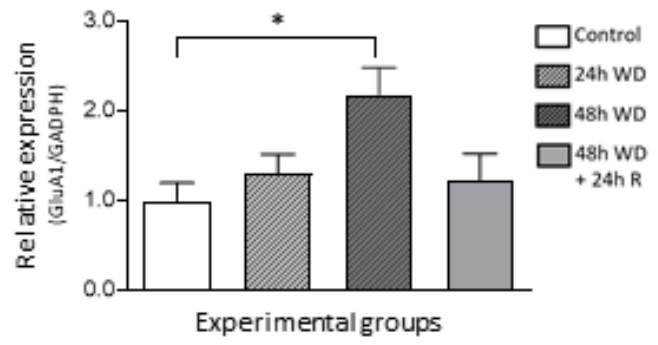

C5

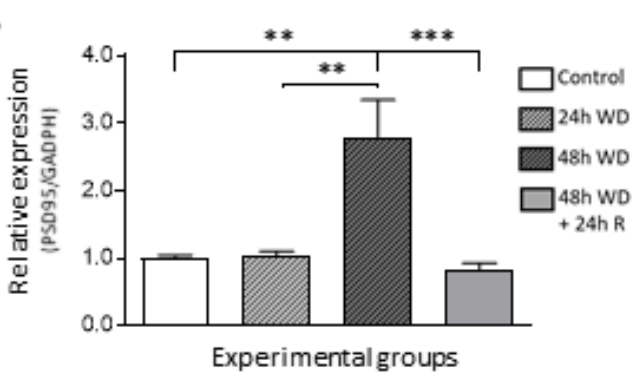

\section{Cortex}

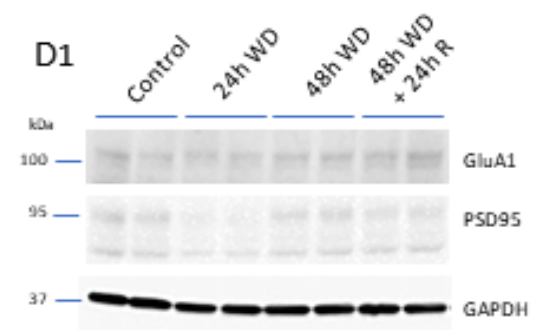

D4

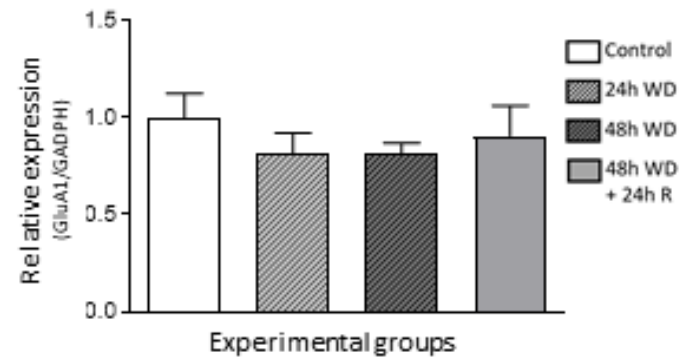

D5
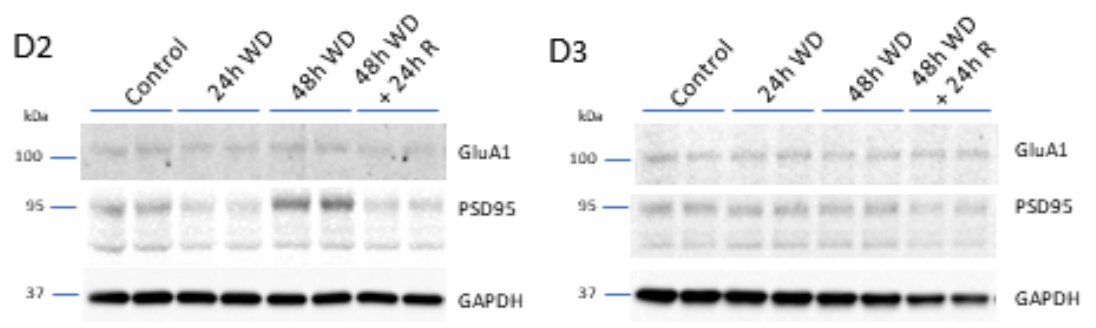


\section{AVP modulates PSD protein expressions}

Figure 2. Prolonged water deprivation (WD) effectively increased GluA1 and PSD95 expression in the main AVPMNN's ascending collaterals targeting regions: amygdala $(A)$, lateral habenula $(B)$; ventral hippocampus (C). We used primary visual cortex (V1), in direct AVPMNN projection has not been observed, as negative control of the experiment (D). Sub-panels 1-3 Western blots of expression time course of two postsynaptic density (PSD) proteins, GluA1 and PSD95 in young male rats, as 4 time points: baseline (control), 24 hours of water deprivation ( $24 \mathrm{~h}$ WD), 48h WD, and $24 \mathrm{~h}$ of recovery with water and food ad libitum after 48h WD (48h WD $+24 \mathrm{~h} \mathrm{R}$ ). Three unit-subjects (see the method section) participated this experiment. In order to show the individual variability and the consistence, as well as the direct basis of the statistic differences, we chose here to show the 3 complete blots that each one includes the complete 4 time-point in duplicates. The primary antibody used for PSD95 here was from UC Davis, clone K28/74, $1 \mathrm{mg} / \mathrm{mL}, 1: 1000$, CA, USA, http://neuromab.ucdavis.edu/datasheet/K28_43.pdf. Sub-panels $\mathbf{A 4}$ and $\mathbf{A 5}$ show the relative expression of GluR1 or PSD-95 respectively in amygdala, relative to the quantity of GAPDH (averages of duplicates of control for each column was assigned as " 1 " and the values were then normalized). A one-way ANOVA showed that treatment significantly affected the expression of GluA1 $(F(3,20)=10.09, p<0.001)$ and PSD-95 $(F(3,20)=17.70, p<0.0001)$. Tukey multiple comparison test showed significantly higher levels of GluA1 after 48h WD (1.600 \pm 0.129$)$ compared to control (1.00 \pm $0.025), 24 h$ WD $(0.983 \pm 0.079)$, and 48h WD $+24 R(0.716 \pm 0.177)$. Expression of PSD95 was also significantly higher in $48 \mathrm{~h}$ WD $(2.067 \pm 0.253)$ compared to control $(1.00 \pm 0.025)$; $24 \mathrm{~h}$ WD $(0.8 \pm$ $0.152)$ or $48 \mathrm{~h}+\mathrm{R}(0.6167 \pm 0.083)$. Sub-panels B4 and B5: show the relative expression levels for GluA1 and PSD95 in lateral habenula (LHb). One-way ANOVA: GluA1 $(F(3,20)=2.216, p<0.117)$, PSD95 (F $(3,20)=7.063, p<0.002)$; PSD95 expression levels: $48 \mathrm{~h}$ WD $(2.805 \pm 0.581)$, control $(1.000 \pm 0.020)$, 24h WD (1.252 \pm 0.202$), 48 h$ WD $+24 h R(1.253 \pm 0.087)$. Sub-panels C4 and C5: show the relative expression levels for GluA1 and PSD95 in ventral hippocampus (vHi). One-way ANOVA GluA1 expression $(F(3,20)=4.154, p<0.019)$ and on PSD95 expression $(F(3,20)=10.76, p<0.0002)$. Tukey multiple comparison test showed increased expression of GluA1 $\left(C_{4}\right)$ in $48 \mathrm{~h} \mathrm{WD}(2.188 \pm 0.300)$ when compared to control $(1.00 \pm 0.201)$. In the case of PSD $95\left(C_{5}\right)$, 48h WD $(2.800 \pm 0.549)$ expression levels were significantly higher than control (1.00 \pm 0.044$) ; 24 \mathrm{~h}(1.033 \pm 0.071)$; and 48h WD $+24 \mathrm{~h} \mathrm{R}$ $(0.833 \pm 0.098)$. Sub-panels D4 and D5: show the relative expression levels for GluA1 and PSD95 in visual cortex (V1). One-way ANOVA of the WD treatment in this area did not elicited any effect for GluA1 $(F(3.20)=1.734, p<0.192)$ nor for PSD95 $(F(3.20)=3.514, p<0.034)$. The asterisks depict significant differences with values as follows: ${ }^{*}: p<0.05 ;{ }^{* *}: p<0.01{ }^{* * *}: p<0.001$ and $+: p=0.061$.

\section{PSD95 and GluA1 expression in ventral hippocampus (vHi) slices are increased after incubation with AVP but not with the AVP fragments used in this study}

To assess if the AVPMNN vasopressinergic innervation to the limbic regions assessed above played a prominent role to upregulate the PSD-95 and GluA1 expression levels observed after water deprivation, we performed an in-vitro pharmacological experiment in acute brain ventral hippocampal slices exposed to vasopressin or some customer designed metabolites, since previous 


\section{AVP modulates PSD protein expressions}

studies have suggested that proteolytic digestion of AVP could result fragments with stronger effects within the central nervous system (Burbach and Lebouille 1983, Reijmers, van Ree et al. 1998, Chepkova, Kapai et al. 2001). We selected the ventral hippocampus due to the highest level of AVP immunopositive fibres reported in our previous study and the AVPMNN system was identified as one of the sources (Zhang and Hernandez 2013). Six experimental conditions/groups consisted in this experiment: ACSF, vasopressin (Arterina, 20 I.U. per ml, Pisa) at a $100 \mathrm{nM}$ concentration or with the following customer-designed vasopressin fragments $\mathrm{AVP}_{4-8}, \mathrm{AVP}_{4-9}, \mathrm{AVP}_{5-8}, \mathrm{AVP}_{5-9}(100 \mathrm{nM})$. We obtained vHi slices from six rats. Each rat provided one slice for each condition. After two hours of incubation with these peptides we carried out Western blot analysis of the expression of PSD95 or GluR1 (Figure 3). AVP group showed a significant increase in the amount of both GluR1 and PSD95 (Fig. 3 A1-A3 show the blots from samples $n=3 \times 2$ (slices from 2 rats were pooled), per each experimental group). Quantification of the relative expressions are depicted in figure $B$ and $C$ for GluA1 and PSD-95, respectively. Customer-designed AVP fragments containing the $4-8$ amino acid sequences used here yielded no-conclusive results (see the discussion technical considerations). Statistic results are presented in the figure legend of figure 3.

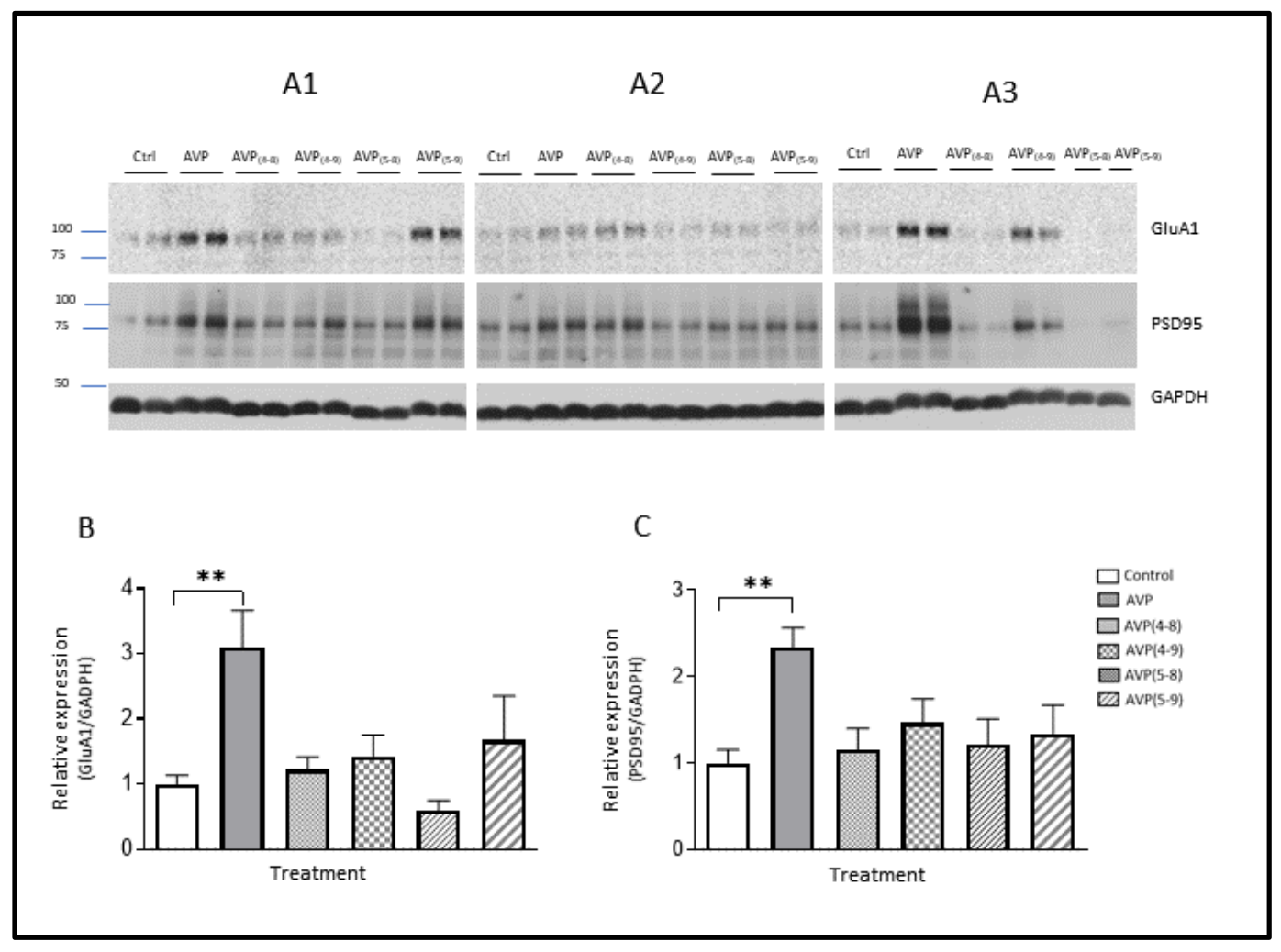

Figure 3. AVP but not the customer-designed AVP fragments evoked the consistent increased expression of GluA1 and PSD95. A1 to A3: GluA1 (upper blot), PSD95 (middle blot) and GAPDH (lower blot) from lysates of ventral hippocampus formation dissected from acute brain slices (thick $300 \mu \mathrm{m}$ ) obtained from 6 rats (two rat samples in each condition, 1 slice each, were pooled), were incubated during two hours in bubbling ACSF (see methods, for details) with commercial vasopressin $100 \mathrm{nM}$ or 


\section{AVP modulates PSD protein expressions}

the peptide fragments $\mathrm{AVP}_{4-8}, \mathrm{AVP}_{4-9}, \mathrm{AVP}_{5-8}$ and $\mathrm{AVP}_{5-9}$ in equimolar concentrations. Panels $\mathrm{B}$ and $\mathrm{C}$ show the quantified relative expression of GluA1 and PSD95, respectively. One-way ANOVA test showed an effect of treatment on the amount of both GluA1 $(F(5,28)=4.999 ; p<0.0021)$ and PSD95 $(F(5.28)=3.672 ; p<0.011)$. To compare between treatment groups, a Tukey multiple comparison test was performed and, in both cases, only AVP elicited a consistent significant increase in the amount of GluA1 (AVP $3.106 \pm 0.562$ vs. Ctrl $1.00 \pm 0.133$ ) and PSD95 (AVP $2.337 \pm 0.226$ vs. Ctrl $1.00 \pm 0.153$ ). Asterisks depict significant differences with values as follows: ${ }^{* *}: p<0.01$

\section{Vasopressin increases the excitability and spine density of pyramidal neurons of ventral hippocampus in ex vivo condition and these effects were blocked by a PI3K inhibitor.}

To investigate if the increased PSD95 and GluA1 levels after incubation with vasopressin could be reflected in electrical excitability changes of the neurons and its PSD expression in ex vivo conditions, we devised the following experiment. First, we assessed the time-course of the effects of AVP in PSD95 and GluA1 expression. Ventral hippocampal sections of $300 \mu \mathrm{m}$ thick were obtained from two PND 30 male rats (figure 4A) and maintained them in four different incubation chambers filled with oxygenated ACSF during 30 minutes in order to let slices stabilize, then we added AVP 100nM (from Professor Manning, $100 \mathrm{nMol}$ AVP) to three of the chambers, control slices were collected immediately. AVP exposed slices were collected after 5 minutes, $1 \mathrm{~h}$ or $2 \mathrm{~h}$. Figure 4B shows that after 1 hour of exposure to AVP there was an increase in the expression levels of GluA1 and PSD95, this increase was maintained at 2 hours. This time course is in accordance with local (dendritic) protein synthesis, which has been addressed as a major factor contributing to the translation of PSD95 mRNA and local protein synthesis, which starts as early as 30 minutes (Friedman, Bresler et al. 2000).

In a second experiment, we evaluated if AVP could increase the excitability of these neurons, thus we prepared brain slices as above mentioned and performed current clamp experiments in pyramidal neurons located in the ventral CA1 area. Figure 4C shows the action potentials elicited by increasing positive current pulses $(50,100,150$ and $200 \mathrm{pA})$ with $500 \mathrm{~ms}$ duration. The upper row (blue) shows a representative trace acquired immediately after the slice was transferred to the recording chamber and was only exposed to ACSF. After baseline recording, AVP (from Prof. Manning's lab), 100nM, was added to the ACSF and recordings from this new condition were acquired between 45min and 1 hour after AVP was added. Red trace is representative of the activity observed under this condition. After $1 \mathrm{~h}$, the exogenous AVP was removed from medium, and the black traces in the lower row represent the response of the neurons to the current injection after AVP wash. Figure 4D shows the average (+/- SEM) firing rate response to current steps for 6 recorded neurons in each condition. Insets show no effect of AVP on the resting membrane potential (RMP, top) nor in the input resistance (IR, bottom). After recordings neurons were fixed and incubated with fluorescent streptavidin to verify the phenotype of the cell. Figure $4 \mathrm{E}$ and $\mathrm{F}$ show two examples of neurons recorded in CA1, the former neuron was only exposed only to ACSF and later was exposed to AVP for $1 \mathrm{~h}$. We observed a higher density of PSD95 puncta within the dendritic spines of the AVP exposed neuron (white arrows in $\mathrm{E}$ and $\mathrm{F}$ ). 
A

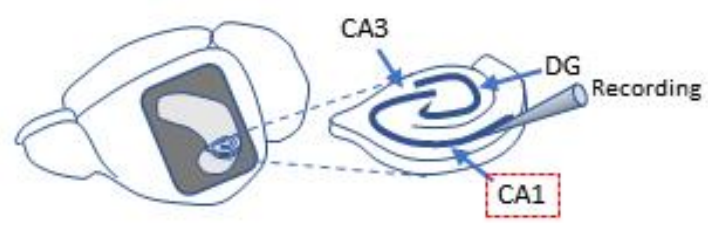

C

${ }_{50}$

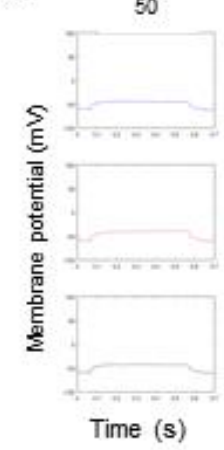

$\mathrm{E}$
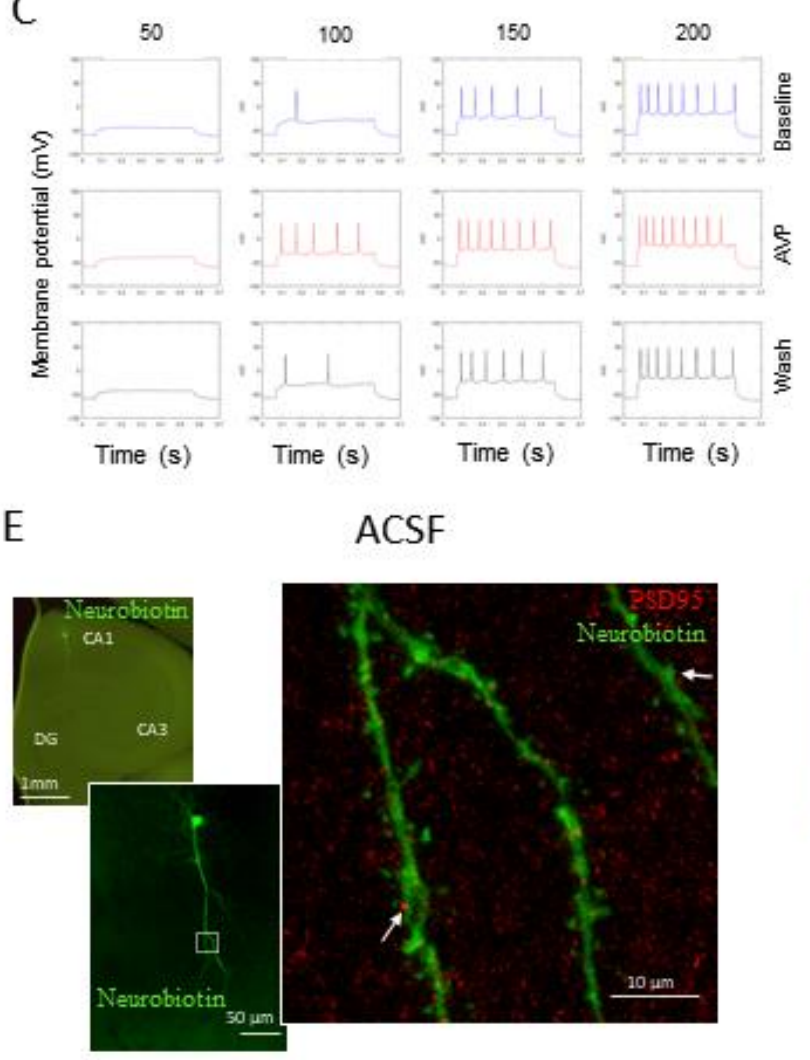

B
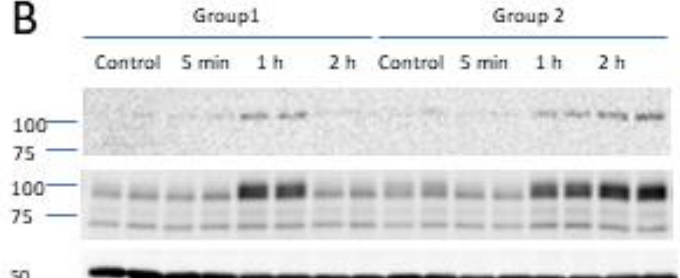

GluA1

PSD95

GAPDH

D
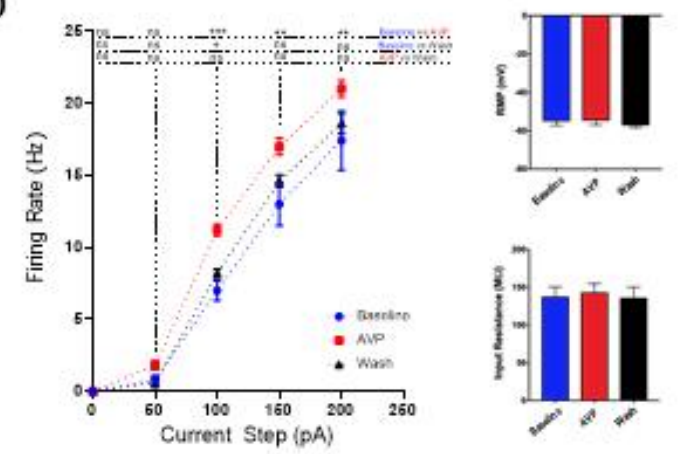

$\mathrm{F}$

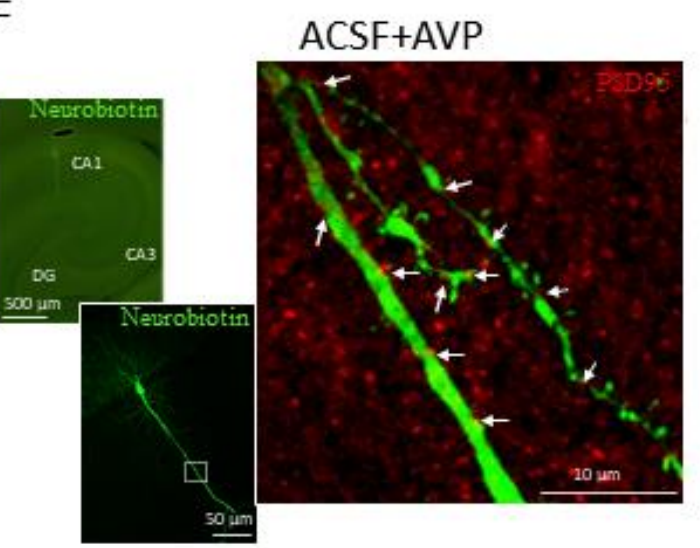

Figure 4. AVP induced increased GluA1 and PSD95 expression in ventral hippocampus is correlated with increased excitability in CA1 pyramidal neurons. A: Schematic representation of the region in $\mathrm{vHi}$, where samples for Western blot assay and acute slice for patch clamp recordings were obtained. CA1 was the region where electrophysiological recordings were made. B: Visible increased levels of expression of PSD95 and GluA1 proteins were observed already at 1 hour after the exposure to 100 nM AVP. C. Traces show the firing rate changes of neurons recorded in CA1 in response to depolarizing 50,100, 150 and 200 pA square 500 ms current injection pulses. The upper row (blue traces) show a representative example of a neuron recorded when only ACSF was present in the medium; red traces show the same cell 45-60 min after AVP 100nMol was added to the bath; and 


\section{AVP modulates PSD protein expressions}

black traces show the recording 30 minutes after the AVP100 nM was washed. D: graph showing the firing rate (average \pm SEM) response to current injection of the 6 neurons recorded for this experiment. A significant increase in the firing rate induced by 100,150 and 200 pA current pulses was observed after AVP was added to the bath. There was a recovery to basal levels after AVP was removed from the medium. No changes in the resting membrane potential (RMP) or Input resistance (IR) were observed. E and F: Confocal photomicrographs of dendrites filled with neurobiotin and processed for PSD95 immunohistochemistry after exposure to ACSF (E) or AVP (F) during $1 \mathrm{~h}$. Notice the increased number of spines in neurons incubated with $100 \mathrm{nM}$ vasopressin, also the higher number of spines that show colocalization with PSD95 (white arrows).

Finally, we investigated the nature of the intracellular pathways associated with AVP regulation of synaptic plasticity in the hippocampus. We incubated hippocampal slices as previously mentioned, but added to the ACSF incubation medium AVP along with a variety of signaling molecules inhibitors: AVP (Kind gift from Professor Manning, 100 nM AVP); AVP + H89 (100 nM AVP + $10 \mu \mathrm{M}$ of the PKA inhibitor); AVP + Go 6976 (100 nM AVP + $10 \mu \mathrm{M}$ of the PKC inhibitor); AVP + U0126 (100 nM $\mathrm{AVP}+10 \mu \mathrm{M}$ of the ERK inhibitor) and AVP + Wm (100 nM AVP $+10 \mu \mathrm{M}$ of the PI3k/Akt inhibitor wortmannin). Following a 60min incubation period, PSD95 levels were assessed by western blot. Inhibition of PKA with H89, PKC with Go 6976, or ERK1/2 with U0126 were without effect on AVPinduced increase in PSD95 levels in hippocampal slices (Figure 5A). Treatment with the PI3K/Akt inhibitor wortmannin did block PSD95 protein induction by AVP (Figure 4A). Thus, we investigated whether or not the excitatory effects of AVP in pyramidal neurons could be abolished by the inhibition of the AKT pathway. Figure 4B shows patch clamp current clamp recordings of neurons that were recorded first with only ACSF in the bath (blue trace). After 45-60 min the medium was changed to provide a concentration of AVP sufficient for neuronal excitation (100 nM; same as Figure 3C) plus a concentration of Wm sufficient to block PSD95 induction by AVP $(10 \mu \mathrm{M})$ (Kirchner, Foehring et al. 2017). Results are shown in the red trace in Figure 4B. Thirty minutes later, AVP and Wm were removed and replaced with ACSF (black trace). Figure $4 C$ shows the I-FR (current - firing rate) curve of the data from 6 recorded slices. Wm blocked the excitatory effect of AVP, with no effect on RMP and IR under this experimental condition.

\section{Discussion}

The synapse is a highly complex subcellular specialization involving closely apposed pre- and post- synaptic membranes and a prominent molecular machinery associated with them that enables chemical-electrical transmission between neurons. Its dynamic adaptation is in response to experience (Somogyi, Tamas et al. 1998). In excitatory post-synapses, a set of molecules is assembled in an ultrastructural feature known as the postsynaptic density (PSD), a narrow electron - dense area subjacent to the postsynaptic membrane (Peters 1970, Boeckers 2006). The PSD is composed of several scaffolding proteins that coordinate the trafficking and membrane insertion of $\alpha$-amino-3hidroxy-5-methyl-4-isxazolepropionate (AMPA)- or N-methyl-D-aspartate (NMDA)-type glutamate ionotropic receptors at the synaptic cleft, thus allowing excitatory transmitter-dependent activation 


\section{AVP modulates PSD protein expressions}

of the postsynaptic neuron and stabilizing synaptic contact (Kennedy 2000, Ehrlich and Malinow 2004, Uchino, Wada et al. 2006, Ehrlich, Klein et al. 2007, Kim, Futai et al. 2007, Taft and Turrigiano 2014, Coley and Gao 2019). This dynamic process appears to be finely tuned by various cues that include autocrine, paracrine and neurocrine signals occurring at the synaptic cleft or in the vicinity of the synapse, that can modulate molecular pathways within the PSD allowing short- and long- term regulation of synaptic plasticity (Rodrigues, Schafe et al. 2004, Park, Rhee et al. 2017).

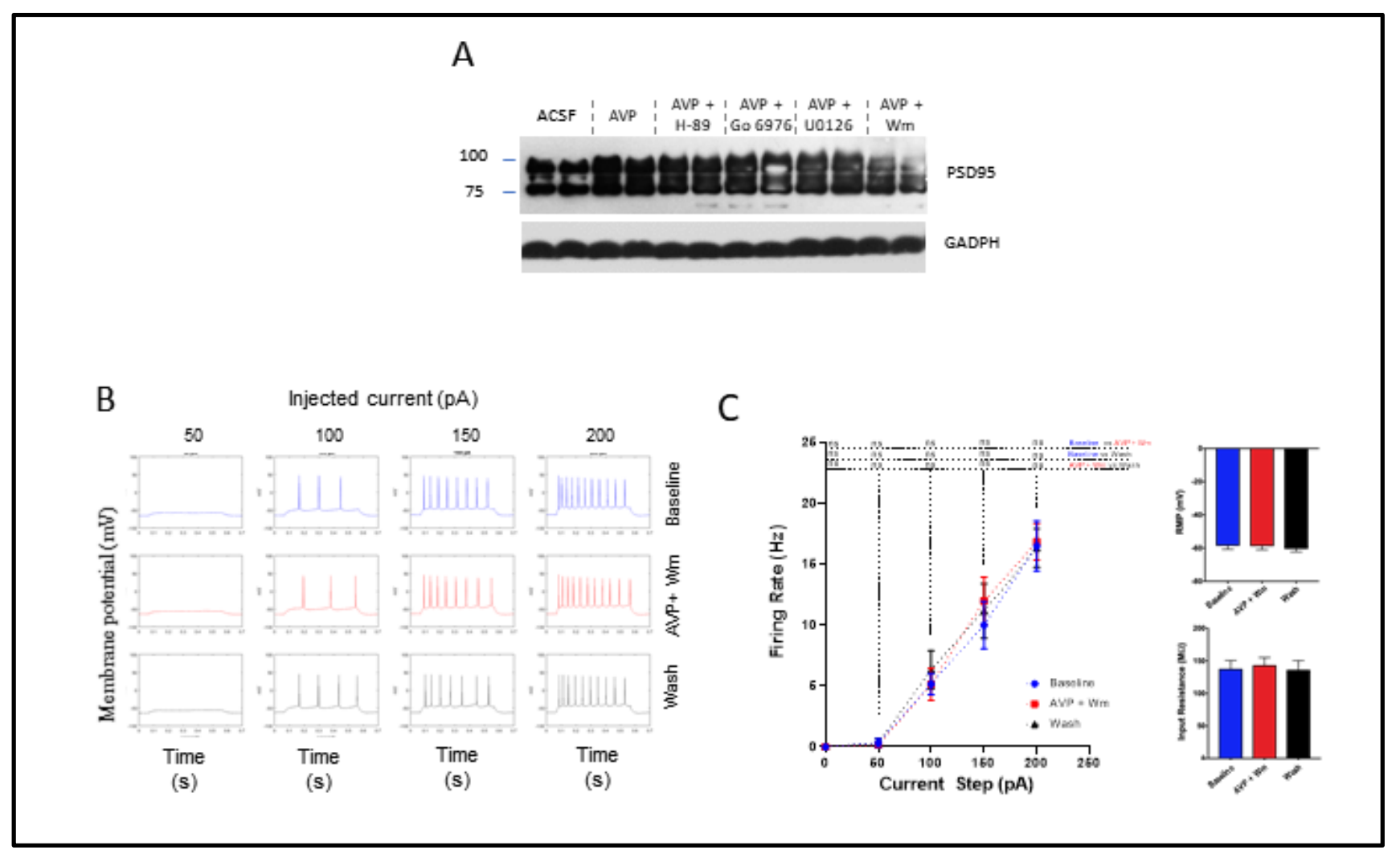

Figure 5. AVP induced increased GluA1 and PSD95 expression and enhanced excitability could be mediated via PI3K / AKT pathway. A: Western blots of samples after application on acute vHi slices with exogenous AVP (100 nM), or AVP (100nM) plus inhibitors H89, Go 6976, U0126 and wortmannin. B: whole cell patch clamp recordings in response to injection of 50, 100150 and 200 pA square 500ms current pulses of neurons in CA1. Blue traces show responses when the neurons were recorded with only ACSF bath; red traces show the response of the same neuron $45 \mathrm{~min}$ after bath was changed to $10 \mu \mathrm{M}$ wortmannin $(\mathrm{Wm})+100 \mathrm{nM}$ AVP. Black trace shows the responses 30 min after AVP and Wm were removed from bath. C: curves show the mean \pm SEM of firing rate response to current injection of 6 neurons recorded with this protocol. Notice that Wm effectively blocked the increased excitability CA1 pyramidal neurons with exogenous AVP application observed in Fig. 4C and 4D. No effect on rest membrane (RMP) nor on input resistance (IR) were observed. 


\section{AVP modulates PSD protein expressions}

We chose to investigate the expression of the glutamate AMPAR receptor subunit GluA1, a molecule which has been reported to be mainly localized within glutamatergic synapses and is hence an excitatory synapse marker (Diering and Huganir 2018), and PSD95, a membrane-associated guanylate kinase, both of which are crucial regulators of postsynaptic organization and function. We focused on limbic projections domains of AVPMNS (Cui, Gerfen et al. 2013, Zhang and Hernandez 2013, Hernandez, Vazquez-Juarez et al. 2015, Hernandez, Hernandez et al. 2016, Zhang, Hernandez et al. 2016), in comparison with non-AVP-innervated brain areas, and under conditions in which the AVPMN system is dramatically upregulated (Dunn, Brennan et al. 1973, Zhang, Medina et al. 2010).

We developed an approach to assess the potential involvement of AVPMNNs in expression of GluA1 and PSD95, in regions of rat limbic system to which these neurons project and at which AVP synapses exist (Zhang and Hernandez 2013, Hernandez, Hernandez et al. 2016, Zhang 2016). Water deprivation, which activates these neurons and increases locomotor motivation-associated behaviors (Zhang, Hernandez et al. 2016), causes prompt enhancement of PSD95 and GluA1 levels in limbic brain areas to which AVP-glutamatergic neurons project, namely amygdala, hippocampus and habenula (Zhang and Hernandez 2013, Hernandez, Hernandez et al. 2016, Zhang, Hernandez et al. 2016) but not in primary visual cortex, an area in which expression of these proteins is equally abundant, but which do not receive AVPergic innervation. Augmentation of PSD95 and GluA1 was reversible upon re-institution of water availability, indicating the state-dependency of this effect. These data reveal a novel role for AVP as a synapse organizer in limbic regions innervated by AVPMNNs, a potential mechanistic link between homeostatic adversity (water deprivation) and motivational drive.

Here, we explored PSD95 and GluA1 expression in AVP-innervated brain regions at various times after water deprivation. Previously we have shown that water-deprived animals exposed to cat odor show diminished freezing behavior and reduced functional output of lateral habenula, likely due to selective activation in GABAergic interneurons in this region (Zhang, Hernandez et al. 2016). This cell population has also been demonstrated to receive monosynaptic input from AVPMNNs (Zhang, Hernandez et al. 2008, Hernandez, Vazquez-Juarez et al. 2015) suggesting that the vasopressinergic system could act as a modulator of synaptic transmission during stress responses. This modulation could well be of physiological importance; in influencing decision making leading to behavioral responses that facilitate survival in the face of homeostatic and environmental threat.

We sought to further expand mechanistic insight into AVP modulation of limbic synaptic dynamics by examining the direct effect of AVP on PSD95 and GluA1 expression, and neuronal excitability, within the ventral hippocampus in an ex vivo slice preparation. Here, application of AVP increased neuronal excitability of pyramidal neurons, and concomitantly enhanced neuronal excitability, within a period of a few hours. The dramatically more rapid effect of AVP ex vivo, compared to water deprivation in vivo, likely reflects the time required to exert up-regulation of AVPergic transmission after water deprivation. 


\section{AVP modulates PSD protein expressions}

The synaptic signaling mechanisms through which AVP exerts its effects on neuronal excitability throughout the brain, acting via engagement with V1 receptors linked to activation of phospholipase $C$, calcium elevation, protein kinase $C$, and protein kinase $B$ (Akt), have been studied extensively (Mizuno, Oomura et al. 1984, Rood and Beck 2014). The effects of neurotrophins and other neuromodulators on PSD-95 transport to the postsynapse, and its expression there, have also been examined in some detail. It has been previously reported that cytoplasmic (dendritic) synthesis of PSD95 and its transport to dendrites can involve signaling through several signaling pathways, including PI3K, ERK, and others (Akama and McEwen 2003, Yoshii and Constantine-Paton 2007) (Yoshii and Constantine-Paton 2014). In our hippocampal slice preparation, we were able to examine the pharmacological profile of signaling generated by AVP exposure on expression of PSD-95, in parallel with the effects of AVP on pyramidal cell excitability. Both were attenuated by treatment with the PI3K inhibitor wortmannin, but not by inhibition of protein kinases $A$ or $C$, implying the involvement of a Gq-linked signaling pathway in both events.

Besides the possibility of AVP directly activating the Akt pathway via Gq signaling, an additional explanation for the effects of AVP on PSD95 expression could be by an indirect effect through brain derived neurotrophic factor (BDNF) signaling. It has been shown that activation of BDNF receptor trough the TrkB/PIP3K/AKT pathway regulates local protein synthesis at synapses in the hippocampus (Leal, Comprido et al. 2014) and that the localization of PSD95 can be regulated by pathways downstream of TrkB signaling (Yoshii and Constantine-Paton 2014).

It is recognized that the hypothalamus is a master hub that integrates metabolic signals with other centers of the brain involved in cognitive and emotional functions. We have demonstrated that AVPMNNs synaptically innervate the hippocampus (Zhang and Hernandez 2013), and that vasopressinergic system influences the performance of a hippocampal-dependent task (Hernandez, Ruiz-Velazco et al. 2012). It has also been shown that under salt loading conditions, BDNF is produced in the supraoptic magnocellular neurons (Balapattabi, Little et al. 2018). In the hypothalamus BDNF increase the excitability of magnocellular neurons and vasopressin release in response to salt intake (Choe, Han et al. 2015).

Increase in dendritic spines on CA1 pyramidal neurons after treatment of hippocampal slices ex vivo with AVP is an intriguing observation: we do not know if increased spine density drives, or is driven by, increased PSD-95 and/or GluA1 expression induced by AVP.

In conclusion, we present a series of experiments, conducted in vivo and ex vivo, that in aggregate demonstrate a potential role for the neuropeptide vasopressin as a synapse organizer acting at multiple levels, via phosphatidyl inositide signaling, to dynamically alter synaptic structure and function in the limbic system. Multiple avenues for further mechanistic investigations are opened up by these observations. Those include i) the downstream pathways emanating from Gq-dependent signaling that result in synaptic protein regulation; ii) the nature of the dramatic up-regulation of PSD- 


\section{AVP modulates PSD protein expressions}

95 and Glu1A protein levels at transcriptional, translational, and post-translational loci; iii) the mechanisms of the prompt reversal of these effects (bidirectional plasticity) to create a flexible platform for future homeostatic and environmental challenges.

\section{$\underline{\text { Technical consideration }}$}

The absence of consistent effects of the AVP-fragments used in this study for inducing the expression of PSD95 and GluA1 deserves further investigation. The fragments we used were customer-designed and synthesized without adding a pyroglutamic residue, which naturally occurs when AVP is metabolized in the brain, blocking the effect of aminopeptidases, increasing its half-life (Burbach, Schoots et al. 1998). This observation has been strengthened by other studies, which artificially added a cysteinyl methyl ester derivative that increases its potency almost forty times higher than AVP, probably by blocking the elimination of the peptide fragments (Lin, Liu et al. 1990). Our negative results from this study suggest that these naturally or artificially biochemical modifications of AVP fragments (cyclization of pyroglutamine) or esterification may be crucial for their previously reported effects in the brain.

\section{Acknowledgements}

We thank Prof. Maurice Manning for his kind gift of arginine vasopressin and Dr. María del Carmen Cardenas-Aguayo for helping with Western blot experiments. MAZ thanks CONACYT postdoctoral fellowship through CONACYT-CB-238744. TPF and ECL were supported by CONACYT PhD scholarship (\#289190) and through grant CONACYT-CB-238744. This study was supported by grants: UNAMDGAPA-PAPIIT-IN216918 \& CONACYT-CB-238744.

\section{References}

Akama, K. T. and B. S. McEwen (2003). "Estrogen stimulates postsynaptic density-95 rapid protein synthesis via the Akt/protein kinase B pathway." J Neurosci 23(6): 2333-2339.

Balapattabi, K., J. T. Little, G. E. Farmer and J. T. Cunningham (2018). "High salt loading increases brain derived neurotrophic factor in supraoptic vasopressin neurones." J Neuroendocrinol 30(11): e12639.

Bargmann, W. and E. Scharrer (1951). "The site of origin of the hormones of the posterior pituitary." Am Sci 39(2): 255-259.

Boeckers, T. M. (2006). "The postsynaptic density." Cell Tissue Res 326(2): 409-422.

Boone, M. and P. M. Deen (2008). "Physiology and pathophysiology of the vasopressin-regulated renal water reabsorption." Pflugers Arch 456(6): 1005-1024.

Brown, C. H., M. Ludwig, J. G. Tasker and J. E. Stern (2020). "Somato-dendritic vasopressin and oxytocin secretion in endocrine and autonomic regulation." J Neuroendocrinol 32(6): e12856.

Burbach, J. P. and J. L. Lebouille (1983). "Proteolytic conversion of arginine-vasopressin and oxytocin by brain synaptic membranes. Characterization of formed peptides and mechanisms of proteolysis." J Biol Chem 258(3): 1487-1494.

Burbach, J. P., O. Schoots and F. Hernando (1998). "Biochemistry of vasopressin fragments." Prog Brain Res 119: 127-136. 


\section{AVP modulates PSD protein expressions}

Chen, C., R. D. Diaz Brinton, T. J. Shors and R. F. Thompson (1993). "Vasopressin induction of long-lasting potentiation of synaptic transmission in the dentate gyrus." Hippocampus 3(2): 193-203.

Chepkova, A. N., N. A. Kapai and V. G. Skrebitskii (2001). "Arginine vasopressin fragment AVP(4-9)facilitates induction of long-term potentiation in the hippocampus." Bull Exp Biol Med 131(2): 136-138.

Choe, K. Y., S. Y. Han, P. Gaub, B. Shell, D. L. Voisin, B. A. Knapp, P. A. Barker, C. H. Brown, J. T. Cunningham and C. W. Bourque (2015). "High salt intake increases blood pressure via BDNF-mediated downregulation of KCC2 and impaired baroreflex inhibition of vasopressin neurons." Neuron 85(3): 549-560.

Coley, A. A. and W. J. Gao (2019). "PSD-95 deficiency disrupts PFC-associated function and behavior during neurodevelopment." Sci Rep 9(1): 9486.

Cui, Z., C. R. Gerfen and W. S. Young, 3rd (2013). "Hypothalamic and other connections with dorsal CA2 area of the mouse hippocampus." J Comp Neurol 521(8): 1844-1866.

De Wied, D. (1971). "Long term effect of vasopressin on the maintenance of a conditioned avoidance response in rats." Nature 232(5305): 58-60.

de Wied, D. (1976). "Behavioral effects of intraventricularly administered vasopressin and vasopressin fragments." Life Sci 19(5): 685-690.

Diering, G. H. and R. L. Huganir (2018). "The AMPA Receptor Code of Synaptic Plasticity." Neuron 100(2): 314329.

Dubrovsky, B., A. Tatarinov, K. Gijsbers, J. Harris and A. Tsiodras (2003). "Effects of arginine-vasopressin (AVP) on long-term potentiation in intact anesthetized rats." Brain Res Bull 59(6): 467-472.

Dunn, F. L., T. J. Brennan, A. E. Nelson and G. L. Robertson (1973). "The role of blood osmolality and volume in regulating vasopressin secretion in the rat." J Clin Invest 52(12): 3212-3219.

Ehrlich, I., M. Klein, S. Rumpel and R. Malinow (2007). "PSD-95 is required for activity-driven synapse stabilization." Proc Natl Acad Sci U S A 104(10): 4176-4181.

Ehrlich, I. and R. Malinow (2004). "Postsynaptic density 95 controls AMPA receptor incorporation during long-term potentiation and experience-driven synaptic plasticity." J Neurosci 24(4): 916-927.

Friedman, H. V., T. Bresler, C. C. Garner and N. E. Ziv (2000). "Assembly of new individual excitatory synapses: time course and temporal order of synaptic molecule recruitment." Neuron 27(1): 57-69.

Hernandez, V., E. Vazquez-Juarez, M. MM, J. H. F, R. Barrio and L. Zhang (2015). "Extra-neurohypophyseal axonal projections from individual vasopressin-containing magnocellular neurons in rat hypothalamus." Frontier in Neuroanatomy.

Hernandez, V. S., O. R. Hernandez, M. Perez de la Mora, M. J. Gomora, K. Fuxe, L. E. Eiden and L. Zhang (2016). "Hypothalamic Vasopressinergic Projections Innervate Central Amygdala GABAergic Neurons: Implications for Anxiety and Stress Coping." Front Neural Circuits 10: 92.

Hernandez, V. S., S. Ruiz-Velazco and L. Zhang (2012). "Differential effects of osmotic and SSR149415 challenges in maternally separated and control rats: the role of vasopressin on spatial learning." Neurosci Lett 528(2): 143-147.

Hernandez, V. S., E. Vazquez-Juarez, M. M. Marquez, F. Jauregui-Huerta, R. A. Barrio and L. Zhang (2015). "Extra-neurohypophyseal axonal projections from individual vasopressin-containing magnocellular neurons in rat hypothalamus." Front Neuroanat 9: 130.

Kennedy, M. B. (2000). "Signal-processing machines at the postsynaptic density." Science 290(5492): 750 754.

Kim, M. J., K. Futai, J. Jo, Y. Hayashi, K. Cho and M. Sheng (2007). "Synaptic accumulation of PSD-95 and synaptic function regulated by phosphorylation of serine-295 of PSD-95." Neuron 56(3): 488-502.

Kirchner, M. K., R. C. Foehring, L. Wang, G. K. Chandaka, J. C. Callaway and W. E. Armstrong (2017). "Phosphatidylinositol 4,5-bisphosphate (PIP2) modulates afterhyperpolarizations in oxytocin neurons of the supraoptic nucleus." J Physiol 595(14): 4927-4946.

Leal, G., D. Comprido and C. B. Duarte (2014). "BDNF-induced local protein synthesis and synaptic plasticity." Neuropharmacology 76 Pt C: 639-656. 


\section{AVP modulates PSD protein expressions}

Lin, C., R. Y. Liu and Y. C. Du (1990). "Cysteinyl methyl ester of AVP(4-8), a potent agonist on the maintenance of passive avoidance in rats." Peptides 11(4): 633-639.

Mizuno, Y., Y. Oomura, N. Hori and D. O. Carpenter (1984). "Action of vasopressin on CA1 pyramidal neurons in rat hippocampal slices." Brain Res 309(2): 241-246.

Mruk, D. D. and C. Y. Cheng (2011). "Enhanced chemiluminescence (ECL) for routine immunoblotting: An inexpensive alternative to commercially available kits." Spermatogenesis 1(2): 121-122.

Muhlethaler, M., J. J. Dreifuss and B. H. Gahwiler (1982). "Vasopressin excites hippocampal neurones." Nature 296(5859): 749-751.

Park, H., J. Rhee, K. Park, J. S. Han, R. Malinow and C. Chung (2017). "Exposure to Stressors Facilitates LongTerm Synaptic Potentiation in the Lateral Habenula." J Neurosci 37(25): 6021-6030.

Peters, A. (1970). The fine structure of the nervious system. New York City, U. S. , Harper Row.

Pow, D. V. and J. F. Morris (1989). "Dendrites of hypothalamic magnocellular neurons release neurohypophysial peptides by exocytosis." Neuroscience 32(2): 435-439.

Reijmers, L. G., J. M. van Ree, B. M. Spruijt, J. P. Burbach and D. De Wied (1998). "Vasopressin metabolites: a link between vasopressin and memory?" Prog Brain Res 119: 523-535.

Rodrigues, S. M., G. E. Schafe and J. E. LeDoux (2004). "Molecular mechanisms underlying emotional learning and memory in the lateral amygdala." Neuron 44(1): 75-91.

Rood, B. D. and S. G. Beck (2014). "Vasopressin indirectly excites dorsal raphe serotonin neurons through activation of the vasopressin1A receptor." Neuroscience 260: 205-216.

Somogyi, P., G. Tamas, R. Lujan and E. H. Buhl (1998). "Salient features of synaptic organisation in the cerebral cortex." Brain Res Brain Res Rev 26(2-3): 113-135.

Taft, C. E. and G. G. Turrigiano (2014). "PSD-95 promotes the stabilization of young synaptic contacts." Philos Trans R Soc Lond B Biol Sci 369(1633): 20130134.

Uchino, S., H. Wada, S. Honda, Y. Nakamura, Y. Ondo, T. Uchiyama, M. Tsutsumi, E. Suzuki, T. Hirasawa and S. Kohsaka (2006). "Direct interaction of post-synaptic density-95/DIg/ZO-1 domain-containing synaptic molecule Shank3 with GluR1 alpha-amino-3-hydroxy-5-methyl-4-isoxazole propionic acid receptor." J Neurochem 97(4): 1203-1214.

Urban, I. J. (1998). "Effects of vasopressin and related peptides on neurons of the rat lateral septum and ventral hippocampus." Prog Brain Res 119: 285-310.

Yoshii, A. and M. Constantine-Paton (2007). "BDNF induces transport of PSD-95 to dendrites through PI3K-

AKT signaling after NMDA receptor activation." Nat Neurosci 10(6): 702-711.

Yoshii, A. and M. Constantine-Paton (2014). "Postsynaptic localization of PSD-95 is regulated by all three pathways downstream of TrkB signaling." Front Synaptic Neurosci 6: 6.

Zhang, L. and L. E. Eiden (2019). "Two ancient neuropeptides, PACAP and AVP, modulate motivated behavior at synapses in the extrahypothalamic brain: a study in contrast." Cell Tissue Res 375(1): 103-122.

Zhang, L., Hernadez, V.S, Vazquez-Juárez, E., Chay, F.K., Barrio, R.A (2016). "Thirst is associated with suppression of habenula output and active stress coping: Is there a role for a non-canonical vasopressinglutamate pathway?".

Zhang, L. and V. S. Hernandez (2013). "Synaptic innervation to rat hippocampus by vasopressin-immunopositive fibres from the hypothalamic supraoptic and paraventricular nuclei." Neuroscience 228: 139-162. Zhang, L., V. S. Hernandez, M. Medina-Pizarro, P. Valle-Leija, A. Vega-Gonzalez and T. Morales (2008). "Maternal hyperthyroidism in rats impairs stress coping of adult offspring." J Neurosci Res 86(6): 1306-1315. Zhang, L., V. S. Hernandez, J. D. Swinny, A. K. Verma, T. Giesecke, A. C. Emery, K. Mutig, L. M. Garcia-Segura and L. E. Eiden (2018). "A GABAergic cell type in the lateral habenula links hypothalamic homeostatic and midbrain motivation circuits with sex steroid signaling." Transl Psychiatry 8(1): 50.

Zhang, L., V. S. Hernandez, E. Vazquez-Juarez, F. K. Chay and R. A. Barrio (2016). "Thirst Is Associated with Suppression of Habenula Output and Active Stress Coping: Is there a Role for a Non-canonical VasopressinGlutamate Pathway?" Front Neural Circuits 10: 13. 
bioRxiv preprint doi: https://doi.org/10.1101/2020.11.07.373027; this version posted November 10, 2020. The copyright holder for this preprint (which was not certified by peer review) is the author/funder. All rights reserved. No reuse allowed without permission.

\section{AVP modulates PSD protein expressions}

Zhang, L., V. S. Hernandez, M. A. Zetter and L. E. Eiden (2020). "VGLUT-VGAT expression delineates functionally specialised populations of vasopressin-containing neurones including a glutamatergic perforant path-projecting cell group to the hippocampus in rat and mouse brain." J Neuroendocrinol 32(4): e12831. Zhang, L., M. P. Medina, V. S. Hernandez, F. S. Estrada and A. Vega-Gonzalez (2010). "Vasopressinergic network abnormalities potentiate conditioned anxious state of rats subjected to maternal hyperthyroidism." Neuroscience 168(2): 416-428. 